\title{
LA BOLIVIE ET L'INDIEN AUTORISÉ
}

\author{
Norbert Rouland*
}

\begin{tabular}{l|l}
\hline RECEBIDO EM: & 18.4 .2020 \\
\hline APROVADO EM: & $\mathbf{3 0 . 4 . 2 0 2 0}$ \\
\hline
\end{tabular}

* Norbert Rouland: Doutor em Direito (Direito Romano), em Ciência Política e em Antropologia Jurídica. Professor concursado no Institut Universitaire de France, onde fundou a cátedra de antropologia jurídica. Diretor do "Master 2" em de Direito das Atividades Artísticas (Droit des activités artistiques) e Professor emérito da Faculté de Droit et Science Politique Aix-Marseille. E-mail: norbert. rouland@wanadoo.fr 
- NORBERT ROULAND

- RÉSUMÉ : L'arrivée au pouvoir en 2005 d'Evo Morales avait suscité de grands espoirs chez les autochtones, fort nombreux en Bolivie. Mais sa présidence a déçu dans un Etat officiellement multiculturaliste. L'article étudie brièvement le multiculturalisme en Amérique latine. Il s'attache aussi à montrer que l'idée de l'Indien sur laquelle se fondent les politiques multiculturelles boliviennes diffère souvent de la réalité. L'Indien ne peut être que rural ; alors que les Indiens émigrent de plus en plus vers les villes et s'y installent. D'autre part, l'article étudie le fonctionnement des justices autochtones et les cas d'incompatibilité de leurs décisions avec les droits de l'homme. Il conclut sur l'idée que la représentation du Bon Sauvage léguée par le XVIIIe siècle est toujours présente, alors que les autochtones décident souvent eux-mêmes d'entrée dans la modernité.

- mots-CLÉs : multiculturalisme. Justices autochtones. Droits de l'homme. Amérique Latine.

\section{BOLIVIA E OS ÍNDIOS AUTORIZADOS}

- Resumo: Com a chegada de Evo Morales ao poder em 2005, houve grandes esperanças entre os índios nativos na Bolívia. No entanto, seu governo representou uma decepção diante de um Estado oficialmente multiculturalista. Este artigo analisa de forma breve o multiculturalismo na América Latina. Procura também mostrar que a ideia do índio em que se baseiam as políticas multiculturais bolivianas difere frequentemente da realidade. $\bigcirc$ índio só pode ser do campo, em que pese a migração cada vez mais frequente dos índios para cidade, com a intenção de nela permanecerem. Por um outro lado, o artigo examina o funcionamento da justiça para os povos indígenas e os casos de incompatibilidade de suas decisões com os Direitos Humanos. A conclusão do artigo gira em torno da ideia de que a representação do "Bom Selvagem" divulgada no século XVIII, ainda está presente, enquanto os povos indígenas decidem muitas vezes por si próprios entrar na modernidade.

- Palavras-chave: Multiculturalismo. Justiça nativa. Direitos do Homem. América Latina. 


\section{BOLIVIA AND INDIAN ALLOWED}

- ABSTRACT: The arrival in power in 2005 of Evo Morales had raised great hopes among the indigenous people, of whom there were many in Bolivia. But his presidency disappointed in an officially multiculturalist state. This article briefly examines multiculturalism in Latin America. It also tries to show that the idea of the Indian on which Bolivian multicultural policies are based often differs from reality. The Indian can only be a rural person; whereas Indians are increasingly migrating to and settling in cities. On the other hand, the article examines the functioning of indigenous justice and the cases of incompatibility of their decisions with human rights. It concludes with the idea that the representation of the "Bon Sauvage» bequeathed by the eighteenth century is still present, while the natives often decide themselves to enter modernity. KEywords: Multiculturalism. Aboriginal Justice. Human Rights. Latin America.

\section{Introduction}

L'actualité internationale récente a été marquée par la destitution tumultueuse d'Evo Morales, Président indien d'un État pluri- national à forte proportion de population autochtone(les deux tiers). Dans un pays acquis officiellement au multiculturalisme, que signifie cette politique adoptée par d'autres Etats d'Amérique du Sud, notamment par rapport à l'instauration d'une justice autochtone ? À quelle image de l'autochtone correspond-elle?

En 2005, son élection est célébrée symboliquement dans les ruines précoloniales de Tiwanaku avant de l'être au Parlement. Son accession à la Présidence de la République entérineles aspirations à la citoyenneté et à l'égalité des Indiens. En Janvier 2006, il annonce que cinq siècles d'oppression allaient être remplacés par cinq nouveaux siècles où prévaudraient les valeurs et les normes autochtones. La Constitution de 2009 , approuvée par référendum avec $59 \%$ de votes positifs, s’inscrit en faux contre l'image misérabiliste des autochtones. Elle proclame l'égalité civique entre tous les Boliviens, mais institue des régimes d'autonomie pour les 36 groupes d'autochtones du pays. En 2009 le whipala, drapeau des peuples autochtones,devient un symbole 
national. Il sert aussi d'amulette de protection aux véhicules des particuliers et des transports publics ${ }^{1}$. La Bolivie est signataire de la Convention 169 de l'Organisation internationale du travail depuis 1991, un instrument international reconnaissant les droits des peuples autochtones ; une loi bolivienne du 7 novembre 2007 a approuvé la Déclaration des droits des peuples autochtones de l'ONU de la même année. Cependant, en 2019, une part importante des organisations autochtones avait retiré leur soutien au gouvernement estimant que le principe du " pouvoir partagé » (rotation des charges) propre aux peuples autochtones n'était pas respecté. En effet, une loi de 2019 privaitles organisations des peuples autochtones du droit à participer aux élections nationales en tant qu'institutions représentatives, n'autorisant que la participation exclusive des partis politiques.

Dans ses derniers discours Evo Morales ne mentionnait plus la justice propre aux communautés autochtones et insistait même sur l'intégration des valeurs autochtones au sein du système général.

L'économie bolivarienne avait connu une période de croissance lors de la flambée du prix des matières premières, permettant la réduction de la pauvreté. Les autochtones en avaient profité grâce à de nombreux programmes en matière de droits sociaux : principalement la santé et l'éducation.

De même, Evo Morales avait voulu qu'ils puissent participer au bénéfice escompté de l'exploitation des réserves de lithium découvertes dans le Salar d'Uyuni. Pour faire obstacle à la mainmise des multinationales il voulait assurer la confection sur place des batteries au lithium, l'État bolivien restant majoritaire à $51 \%$ dans les accords conclus avec des sociétés étrangères. Le groupe français Bolloré avait été intéressé, mais surtout les Chinois (Xinjianq TBEA Group), qui entendent conserver le quasi-monopole dont ils jouissent sur les terres rares ${ }^{2}$. Mais après le putsch qui a renversé Evo Morales, on peut penser que le gouvernement de droite qui lui succédera préférera la voie des privatisations ${ }^{3}$.

1 Cf. Ivone JUARES, El Pais, Madrid, 18 Novembre 2019.

2 Ils s'intéressent aussi au Groenland : Cf. N. ROULAND, Le Groenland : un eldorado pour le XXIe siècle ? À quel Coût?, Revue de la recherche juridique, 2018-2, XLIII-172, 497-518. Par ailleurs, il existe d'importants gisements de lithium au Tibet.

3 La demande mondiale de lithium était d'environ 300000 t en 2018. Elle devrait atteindre 1 million de tonnes en 2040. En Bolivie, les réserves sont évaluées à 21 millions de tonnes. Cf : Bruce BAGLEY -Olga NAZARIO, Lithium and Bolivia : the Promise and the Problems, Western Hemisphere Security Analysis Center, 53, June 2010 ; Maëlle MARIETTE, Un projet d'industrie nationale torpillée par le coup d'État-En Bolivie, la filière lithium à l'encan, Le Monde Diplomatique, janvier 2020, 23. 
Cependant, ces dernières années, le modèle économique avait commencé à se fissurer : le déficit budgétaire était devenu supérieur à $8 \%$ du PIB en 2018.

Beaucoup d'autochtones, les cholos, ont quitté la campagne et vivent en ville. Ils font partie des classes moyennes. Ils avaient d'abord soutenu Evo Morales, mais ils s'en sont détournés quand celui-ci a engagé la lutte contre l'économie informelle, qui représentait $60 \%$ du PIB et concernait $70 \%$ de la population active il y a une dizaine d'années ${ }^{4}$. De plus, la construction de la pluri nationalité les excluait : l'Indien autorisé est un Indien rural.

Mais il faut insérer le cas de la Bolivie dans le cadre plus général du multiculturalisme.

\section{Sur le concept de multiculturalisme et de justice}

Celui-ci a très mauvaise presse en France, un pays qui ne reconnaît pas sur son sol les minorités et les peuples autochtones ${ }^{5}$; honnit ce que la plupart des médias appelle le communautarisme, considéré comme l'inverse des principes républicains ; n'est pas un État pluri- national ; et dont la jurisprudence du Conseil constitutionnel affirme depuis longtemps qu'il ne peut exister en France de droits collectifs, ni d'autre peuple que le peuple français.

Comme toutes les notions générales, le multi- culturalisme doit être contextualisé ${ }^{6}$. Les principes égalitaires français ne peuvent être appliqués mécaniquement dans des sociétés comportant une part de population autochtone, qu'ils risquent de marginaliser. Né il y a une cinquantaine d'années au Canada, le multiculturalisme s'est par la suite développé aux États-Unis et en Amérique du Sud7. Dans les années 1990 sont

4 Cf. Maëlle MARIETTE, Le Monde Diplomatique, Septembre 2019. Pour une approche plus générale: N. ROULAND, Anthropologie Juridique, Paris, PUF, 1988 ; (dir.) Droit des Minorités et des peuples autochtones, Paris, PUF, 1996 ; Les colonisations juridiques, de l'Arctique à L'Afrique Noire, Journal of Legal Pluralism, 39-135.

5 Ce modèle s'est assoupli en ce qui concerne certains territoires ultra -marins et a donc acquis une géométrie variable, surtout en Nouvelle-Calédonie depuis les accords de 1998. Les Kanaks y jouissent d'un statut personnel, il existe des juridictions coutumières et un droit civil coutumier est en train de se former : cf. Antoine LECA, Précis de droit civil coutumier kanak, Centre de documentation pédagogique de Nouvelle-Calédonie, 2018 ; Régis LAFARGUE, La coutume judiciaire en Nouvelle-Calédonie-Aux sources d'un droit commun coutumier, Aix-en-Provence, Presses universitaires d'Aix-Marseille, 2003.

6 Cf. Will KYMLICKA, La Citoyenneté multiculturelle, une théorie libérale du droit des minorités, Paris, La Découverte, 2001.

7 Cf. Boaventura DE SOUSA SANTOS et José Luis EXENI RODRIGUEZ, Justiciaindigena, plurinationalidad e intercultural en Bolivia, Quito, Fundation Rosa Luxemburg /Abya Yala, 2012. 
intervenues les premières reconnaissances du caractère multiculturel dans les droits des Etats latino- américains. Notamment l'Équateur en 2008 (7,3\% d'autochtones). La Cour Constitutionnelle de Colombie a fait appel à des études anthropologiques en faveur d'une interprétation limitative des droits de l'homme.

Au Venezuela (2,6 \% d'autochtones), la Constitution de 1999 a instauré une justice autochtone.

C'est tout ici le cas de certains Etats occidentaux comme le Canada, la Nouvelle-Zélande et l'Australie. La Chine préfère parler de minorités nationales,terme qui s'applique en fait aux peuples autochtones regroupant environ 1/10 de la population chinoise.

Globalement, l'essor de l'indianité a été stimulé par la mondialisation, la recherche identitaire étant dans bien des Etats une réaction à la globalisation. Il faut noter aussi que l'Amérique latine n'a pas connu de menaces du terrorisme islamiste, qui dans d'autres parties du monde, a inhibé le développement de la pensée sur la diversité et la cohabitation interculturelle. Globalement aussi le débat sur l'instauration de politiques pluri nationales n'a pas porté sur les relations de genre. Alors que les femmes sont fréquemment victimes de violences, beaucoup cherchent moins à accuser leur conjoint violent qu'à lui pardonner en vue d'une réconciliation : les structures mentales résistent.

Le pluralisme n'était pas pour autant ignoré dans des époques antérieures. Pendant la colonisation espagnole, le pouvoir royal reconnaissait une capacité juridique propre aux Indiens. Il recommandait aux juges de tenir compte de leurs coutumes, même si les Indiens étaient considérés comme des êtres humains, mais de nature inférieure. Au XIXe siècle, l'indépendance des pays latino-américains a aboli le double système juridique colonial. En Bolivie, Simon Bolivar fait promulguer des lois se basant sur la propriété privée et des principes d'égalité entre citoyens. Mais l'égalité de droit ne se confond pas avec l'égalité de fait : les Indiens constituent une catégorie inférieure de la population, tenue à l'écart des centres de décision. En 1994, des réformes constitutionnelles avaient fait de la Bolivie une société multiethnique, pluriculturelle, et reconnaissaient les droits de propriété collectifs des autochtones sur leurs terres.

Aujourd'hui, le multiculturalisme repose sur certaines représentations qui sont éloignées de la réalité. Au Primitif s’est substituéle Bon Sauvage, sous la figure contemporaine de l'Indien autorisé par les textes juridiques. 
La tradition est censée perpétuer un modèle trans -historique idéalisé. L'Indien actuel ne peut être que rural. Par opposition à la colonisation, les représentants des peuples autochtones décrivent un monde idéal où l'harmonie régnait, sans conflits.

La justice autochtone est devenue récemment unerevendication centrale des autochtones $^{8}$, un symbole de leur identité.

À la fin des années 2000, la Bolivie et l'Équateur l'ont reconnue dans leurs constitutions. Mais il faut observer qu'en Bolivie, il n'existe pas de mot pour traduire Justice ou Institution judiciaire dans les langues autochtones. Le tribunal n'existait pas dans les communautés andines. On préférait parler de disputes, de fautes et de rétablissement d'un équilibre.

La constitution bolivienne de 2009 ne circonscrit pas l'exercice des justices autochtones à un territoire ou à un groupe humain. Leurs compétences leur permettent d'apprécier des actes dont la répercussion a affecté la communauté indigène. Mais par la suite une loi de 2010 a restreint ces compétences : ne sont sujets aux justices autochtones que les membres d'une nation originaire. Les membres d'une communauté autochtone peuvent être jugés au sein de leur communauté, mais également à l'extérieur, alors que les personnes qui ne sont pas autochtones ne peuvent jamais être jugées par les autorités communautaires, même si elles troublent la vie communautaire locale. De plus, la loi restreint la compétence des juridictions : les infractions aux droits de l'homme, le droit international, les délits de corruption, le droit de propriété, le droit du travail, le droit administratif, le droit fiscal, etc.y échappent.

Que reste-t-il ? La terre, son contrôle et sa gestion, ce qui n'est pas rien. La sorcellerie, fréquente. En général les juridictions étatiques ne veulent pas la connaître, mais les litiges qui résultent de sa pratique peuvent aussi se régler en dehors de toute juridiction. Au Cameroun, la sorcellerie est expressément incriminée dans le code pénal. En Nouvelle-Calédonie il arrive que les juges en tiennent compte dans leurs décisions. Devant les juridictions autochtones, le rôle des avocats-pour un avocat,être autochtone facilite la tâche- est prépondérant. Non seulement ils défendent leurs clients, mais jouent le rôle de conseil pour les juges qui souvent manquent de compétence pour

8 Sur ce thème, cf. surtout la récente thèse d'Elise GADEA, Le pluralisme juridique à l'épreuve des pratiques communautaires en Bolivie-Politique d'administration de la " justice indigène originaire paysanne-», Directeur Franck Poupeau, Université Paris III, Paris, 2020. Voir aussi Christian GROS, Pour une sociologie des populations indiennes et paysannes de l'Amérique latine, Paris, L'Harmattan, 1997 ; James M.Cooper, Legal Pluralism and the Threat to human Rights in the new plurinational State of Bolivia, Washington University Global Studies Law Resort, v. 17, Issue 1, 2018. 
rendre leurs sentences sans violer les droits fondamentaux et les droits de l'homme : ils ne connaissent pas bien les limites, qu'il faut leur expliquer.

Car la compatibilité entre les droits de l'homme et les décisions judiciaires de ces instances est un problème majeur. La Bolivie a souscrit à tous les instruments internationaux prohibant la peine de mort, la torture, et prescrivant le droit à un jugement impartial. Or,en Amérique latine, pour beaucoup de juristes, la coutume reste synonyme de barbarie. Les punitions physiques, et beaucoup plus grave, le lynchage, sont des châtiments qui existaient dans la société préhispanique, mais également aujourd'hui. Contre toute évidence, certains défenseurs des organisations autochtones nient les violences physiques dans le système indigène. Alors même que les acteurs des lynchages revendiquent leurs actes comme étant une pratique juridictionnelle, afin d'éviter la prison.

En 2008, l'ONG Human rights Conference a publié un rapport très critique sur la justice communautaire: " une justice qui décide en lisant les feuilles de coca doit être subordonnée, surveillée et révisée constamment». Le rapport visait expressément le fouet(en pratique en diminution) et le lynchage. L'État bolivien, d'abord réticent, a finalement été sensible à ces critiques. En 2010, la Loi de Délimitation juridictionnelle qualifie de lynchage de «violation des droits de l'homme, il n'est permis dans aucune juridiction et doit être prévenu et sanctionné par l'État pluri national bolivien ». D'autres textes de loi ont pour but de préserver de l'arbitraire de la justice indigène les droits de l'homme, des femmes, des enfants, des personnes âgées et des handicapés.

De manière plus générale on note dans d'autres domaines une certaine idéalisation de la justice autochtone, qu'on oppose trop facilement à la justice étatique.

On la présente comme gratuite et rapide. Ce qu'il faut nuancer.Car dans ces communautés, les mécanismes de dons et de contre dons sont fondamentaux. Dans la pratique, la question de la rétribution des autorités coutumières et du paiement des services juridiques est fréquemment posée. De plus, on note la persistance à l'allongement des conflits dans lesquels l'arbitrage des autorités est remis en cause.

Cette justice est aussi présentée comme orale, par opposition à la justice étatique bureaucratique. Dans la pratique, les décisions des autorités coutumières s'accompagnent souvent de la tenue d'un registre écrit avec l'émission de documents écrits, qui servent de preuve. Et les écrits sont attestés même à l'époque pré- hispanique en tant que supports d'arbitrage ou de conflits communautaires.

Dans la vie concrète, les autochtones pratiquent le forum shopping. Ils évaluent de manière empirique le fonctionnement de chaque type de juridiction. La justice éta- 
tique s'exerce à partir d'une loi codifiée. La justice coutumière préfère le cas par cas et le rétablissement de l'harmonie à la détermination précise des responsabilités. Attitude vertueuse, mais parfois peu opératoire.Les autochtonesconsidèrent, non sans raison, que la justice étatique a plus de poids dans l'exécution des décisions. Les autorités coutumières sont jugées peu compétitives par rapport aux juges étatiques, bien formés en droit, et qui disposent de la force coercitive pour l'exécution de leurs décisions. Le principe juridique d'égalité hiérarchique entre la juridiction étatique et la juridiction autochtone est vidé de son sens.

L'échelle des valeurs attribuées aux faits juridiques peut être très différente. Les infractions à caractère religieux peuvent être très graves pour les autochtones, mais légères pour le droit étatique.

\section{Conclusion}

Dans ce qui précède, on voit la force des stéréotypes, un moyen commode de ne pas résoudre les problèmes complexes.

Les peuples autochtones, très divers, aussi bien sur le plan mondial qu'à l'intérieur des Etats, ont une longue histoire qui n'est pas unilinéaire.

En Amérique du Sud, les conquérants espagnols ont été éblouis par la splendeur de certaines cités. Dans les débats autour de l'esclavage des peuples colonisés, on a finalement reconnu aux Indiens une nature humaine, mais de qualité inférieure. Par la suite, du XIXe siècle au milieu du XX ${ }^{\mathrm{e}}$, en Amérique du Sud comme du Nord, on a pensé que les autochtones s'assimileraient aux Européens devenus dominants. À la fin du siècle précédent le droit international a commencé non seulement à constater la persistance de leur existence, mais à leurreconnaître des droits. Ces droits ont été constatés dans les textes constitutionnels et législatifs de plusieurs Etats.

Mais le droit procède toujours de représentations. Et sous les traits de l'Indien autorisé par la loi des Etats, ressuscite le Bon sauvage du XVIIIe siècle. Or certaines pratiques coutumières, nées à une époque où les droits de l'homme n'existaient pas, sont difficilement conciliables avec eux ${ }^{9}$. D’autre part et peut-être surtout, il n'est pas suffisamment tenu compte de l'Histoire. Les coutumes ne sont pas des blocs monolithiques

9 En 1952, dans un discours prononcéà l’Unesco, Claude Lévi-Strauss insistait de façon prémonitoire sur l'incompatibilité possible entre l'universalisme des droits de l'homme et les spécificités culturelles des peuples traditionnels. (Cf. Claude LEVI-STRAUSS, Race et Histoire, Paris, Denoël, 1952, 23). 
se transmettant de génération en génération comme des Écritures saintes juridiques. Elles disparaissent, se transforment, réapparaissent.Enfin, l'anthropologuecède parfois à la tentation de fétichiser le passé, d'opposer lapureté des origines à la corruption des maux de la modernité. Il faut résister à cetteinclination. Même si le temps des diligences a des avantages, qui de nous voudraitréellement y retourner ? D’ailleurs, pour le pire et le meilleur, à de rares exceptions prés, ces peuples veulent la modernité. D’autant plus que grâce aux moyens de communication modernes, ils savent que le monde extérieur existe.

Notre rôle n'est pas de les renvoyer à leur passé et à nos rêves de Bon Sauvage, mais dans la mesure de nos moyens, de les aider à choisir entre leurs avenirs possibles.

\section{RÉFÉRENCES}

BAGLEY, B. ; NAZARIO, O. Lithium and Bolivia: the Promise and the Problems.Western Hemisphere Security Analysis Center, 53, June 2010.

COOPER, J. M. Legal Pluralism and the Threat to human Rights in the new plurinational State of Bolivia. Washington University Global Studies Law Resort, v. 17, issue 1, 2018.

GADEA, E. Le pluralisme juridique à l'épreuve des pratiques communautaires en Bolivie-Politique d'administration de la «justice indigène originaire paysanne ». Thèse, Directeur Franck Poupeau, Université Paris III, Paris, 2020.

GROS, C. Pour une sociologie des populations indiennes et paysannes de l'Amérique latine. Paris : L'Harmattan, 1997.

JUAREZ, I. El Pais, Madrid, 18 Novembre 2019.

KYMLICKA, W. La Citoyenneté multiculturelle, une théorie libérale du droit des minorités. Paris : La Découverte, 2001.

LAFARGUE, R. La coutume judiciaire en Nouvelle-Calédonie-Aux sources d'un droit commun coutumier. Aix-en-Provence : Presses universitaires d'Aix-Marseille, 2003.

LECA, A. Précis de droit civil coutumier kanak. Nouvelle-Calédonie : Centre de documentation pédagogique de Nouvelle-Calédonie, 2018.

LÉVI-STRAUSS, C. Race et Histoire, Paris, Denoël, 1952.

MARIETTE, M. Mérites et limites d’une «révolution» pragmatique. Le Monde Diplomatique, Septembre 2019. Disponible en ligne : https://www.monde-diplomatique.fr /2019/09/MARIETTE/60321

MARIETTE, M. Un projet d'industrie nationale torpillée par le coup d'État-En Bolivie, la filière lithium à l'encan. Le Monde Diplomatique, janvier 2020, p. 23. Disponible en ligne : https://www. monde-diplomatique.fr/2020/01/MARIETTE/61191

ROULAND, N. Anthropologie Juridique. Paris : Presses Universitaires de France, 1988. 
ROULAND, N. Le Groenland : un eldorado pour le XXIe siècle ? À quel Coût ? Revue de la recherche juridique, 2018-2, XLIII-172, p. 497-518.

ROULAND, N. Les colonisations juridiques, de l'Arctique à L'Afrique Noire. Journal of Legal Pluralism, v. 22, p. 39-135, 1990.

ROULAND, N. et al. (dir.) Droit des Minorités et des peuples autochtones. Paris : Presses Universitaires de France, 1996.

SANTOS, B. de S. ; EXENI RODRIGUEZ, J. L. Justiciaindigena, plurinationalidadintercultural en Bolivia : Quito, Fundación Rosa Luxemburg/Abya Yala, 2012. 